\title{
Surface curvature from kinetic depth can affect lightness
}

\author{
Lindsay M. Peterson \\ School of Psychology, UNSW Sydney \\ Daniel J. Kersten \\ Department of Psychology, University of Minnesota \\ Damien J. Mannion \\ School of Psychology, UNSW Sydney
}

\begin{abstract}
The light reaching the eye confounds the proportion of light reflected from surfaces in the environment with their illumination. To achieve constancy in perceived surface reflectance (lightness) across variations in illumination, the visual system must infer the relative contribution of reflectance to the incoming luminance signals. Previous studies have shown that contour and stereo cues to surface shape can affect the lightness of sawtooth luminance profiles. Here, we investigated whether cues to surface shape provided solely by motion (via the kinetic depth effect) can similarly influence lightness. Human observers judged the relative brightness of patches contained within abutting surfaces with identical luminance ramps. We found that the reported brightness differences were significantly lower when the kinetic depth effect supported the impression of curved surfaces, compared to similar conditions without the kinetic depth effect. This demonstrates the capacity of the visual system to use shape from motion to "explain away" alternative interpretations of luminance gradients, and supports the cue-invariance of the interaction between shape and lightness.

- It is useful for our vision to be able to separate how much a surface reflects light from how much light is reaching the surface.

- This study shows that an impression of shape produced by surface movement can affect how we perceive its reflectance.
\end{abstract}

Keywords: vision; lightness; motion; psychophysics; shape

The proportion of light that is reflected from a given surface in the environment is an intrinsic property that is typically stable across time. Knowledge of surface reflectance can thus be useful in performing perceptual tasks such as object recognition (Nayar \& Bolle, 1996; O'Toole, Vetter,

Lindsay M. Peterson, School of Psychology, UNSW Sydney; Daniel J. Kersten, Department of Psychology, University of Minnesota; Damien J. Mannion, School of Psychology, UNSW Sydney.

This research was funded by the Australian Government through the Australian Research Council (DP170100087 to DM and DK) and an Australian Government Research Training Program Scholarship (to LP). We thank Branka Spehar for comments on the manuscript.

Correspondence concerning this article should be addressed to Damien J. Mannion, School of Psychology, UNSW Sydney, NSW, Australia. E-mail: d.mannion@unsw.edu.au

(C) 2018, American Psychological Association. This paper is not the copy of record and may not exactly replicate the final, authoritative version of the article. Please do not copy or cite without authors permission. The final article will be available, upon publication, via its DOI: $10.1037 / x h p 0000575$
\& Blanz, 1999; Russell, Biederman, Nederhouser, \& Sinha, 2007). However, identifying surface reflectance from the luminance reaching the eye requires the visual system to overcome the inherent ambiguity of the retinal image. This ambiguity arises because luminance confounds the reflectance of a surface with its incident illumination, with the result that a given luminance could have been produced by a surface of any reflectance. The challenge for the visual system is to interpret luminance so as to achieve constancy in perceived surface reflectance (known as lightness, in the achromatic domain) across variations in illumination.

Lightness constancy has been investigated using a stimulus formed from two abutting linear luminance gradients (van den Brink \& Keemink, 1976), which produces the sawtooth luminance profile shown in Figure 1A. The two regions with identical luminance gradients typically differ in lightness, similar to the appearance of many in the family of "border effects" (Kingdom \& Moulden, 1988) often referred to as Craik-O'Brien-Cornsweet illusions (Craik, 1966; Cornsweet, 1970; O’Brien, 1958). This appearance has been attributed to the inference that the most probable cause of the luminance profile is two flat surfaces of unequal reflectance under illumination which varies smoothly 



Figure 1. Potential environmental causes of a sawtooth luminance profile. Panel A shows the luminance across the dashed line of an image of a sawtooth luminance profile formed by two abutting surfaces with identical linear luminance ramps. Panel $\mathbf{B}$ shows that the sawtooth luminance profile could be produced by flat surfaces of unequal reflectance under slowly-varying illumination. Panel $\mathbf{C}$ shows that the sawtooth luminance profile could also be produced by curved surfaces of equal reflectance under an illumination with slow and abrupt variations.

across space (Knill \& Kersten, 1991), as depicted in Figure 1B. Because the surfaces in this scenario are of unequal reflectance, our perceptual impression is of correspondingly unequal lightness. Support has been provided for this explanation by demonstrations showing that stimulus configurations that were more compatible with the interpretation of unequal surface reflectance and smoothly-varying illumination tended to produce larger differences in the lightness of the two surfaces (Purves, Shimpi, \& Lotto, 1999).

Importantly, the interpretation of the sawtooth luminance profile can be affected by cues to surface shape. The strength of the lightness difference can be reduced when the surface regions contain curved edge contours, such that the two surfaces are perceived to be of comparable lightness (Knill \&
Kersten, 1991). This cue to surface shape allows the visual system to infer that the luminance profile is caused by changes in illumination as it falls across two curved surfaces of equal reflectance (Knill \& Kersten, 1991), as depicted in Figure 1C. A similar explanation has been proposed to explain the decomposition of luminance gradients (Bergström, 1977), and the perceptual appearance of a 'single sawtooth' profile (a single linear luminance gradient flanked by two regions of uniform intermediate luminance) in which perceiving surface curvature changes the apparent lightness of the flanking regions (van den Brink \& Keemink, 1976).

This dependence of lightness on apparent surface shape can be understood within the concept of "explaining away" (Kersten \& Yuille, 2003; Kersten, Mamassian, \& Yuille, 2004), which involves the competition between two or more alternative hypotheses to account for a certain event (Kersten \& Yuille, 2003; Kersten et al., 2004). In the scenario explored here, and depicted in Figure 1, the hypothesis of a pair of surfaces of unequal reflectance competes with the hypothesis of a pair of surfaces of equal reflectance to account for the sawtooth luminance profile. The contour cue to surface shape provides auxiliary evidence that explains away the hypothesis of a pair of surfaces of unequal reflectance, as the increase in the probability that the surfaces are curved also increases the probability that they are of equal reflectance (Kersten et al., 2004; Kingdom, 2008).

Auxiliary evidence about surface shape can be provided by cues other than the form of the bounding contour, with binocular disparity cues indicative of curved surfaces also producing a reduction in the apparent lightness differences across sawtooth luminance profiles (Buckley, Frisby, \& Freeman, 1994). This suggests that the effects of perceived surface shape on lightness may be cue-invariant, with the visual system able to incorporate auxiliary evidence from various image measurements (Gilchrist, 1977, 1980). However, support for cue-invariance in the interaction between shape and lightness with sawtooth luminance profiles is limited by the range of cues that have been investigated. Currently, only shape from contour (Knill \& Kersten, 1991) and shape from stereo (Buckley et al., 1994) have been considered.

Here, we investigate whether lightness can be influenced by shape information provided by motion. To separate the contribution of motion from other cues, we use the "kinetic depth effect" in which the shape of a object can be identified when moving despite not being apparent during static viewing (Wallach \& O'Connell, 1953). We hypothesise that shape information provided by this kinetic depth is capable of affecting lightness. As discussed, the interaction between shape and lightness may be invariant to the nature of the shape cue given that lightness evoked by a sawtooth luminance profile can be affected by both shape from contour (Knill \& Kersten, 1991) and shape from stereo (Buckley et al., 1994). Furthermore, shape from kinetic depth and 
shape from stereo appear to involve common neural mechanisms (Fang \& He, 2004; Freeman, 1998; Kham \& Blake, 2000; Nieder, 2003); if an interaction with lightness is subsequent to such common processing, the involvement of shape from stereo in lightness (Buckley et al., 1994) predicts that similar effects would be evident with shape from kinetic depth. Finally, shape information from kinetic depth can affect the degree to which a surface is perceived as specular or matte (Marlow \& Anderson, 2016) and can interact with surface transparency (Kersten, Bülthoff, Schwartz, \& Kurtz, 1992) - consistent with shape from kinetic depth being available to support inferences regarding surface material.

In two experiments, we rendered and composited curved surfaces such that the luminance pattern was similar to the conventional sawtooth profile that evokes the perception of unequal lightness across the two surfaces (van den Brink \& Keemink, 1976). The curved surfaces were truncated cylinders in Experiment 1 (Figure 2A-C) and, to increase the strength of the kinetic depth cue, half-cylinders in Experiment 2 (Figure 2D-F). To test the hypothesis that shape from kinetic depth can affect lightness, we also produced images in which the surface reflectance contained texture markings (as shown in Figure 2). During surface motion, such texture markings permitted the perceptual impression of surface curvature. We predicted that this impression would be associated with the two surfaces being perceived as more similar in lightness than when the motion did not produce an impression of a curved surface.

\section{Methods}

\section{Participants}

Participants were recruited from a database of students enrolled in an introductory psychology course and from a database of community volunteer research participants. There were 149 participants in Experiment 1 (115 female, 34 male; median age of 18 years) and 74 participants in Experiment 2 (36 female, 38 male; median age of 19 years). These sample sizes were based on a sequential analysis plan with replacements for excluded participants (described further in the Analysis section). Participants were compensated with course credit or $\$ 15$. Selection criteria for the experiment was self-reported normal or corrected-to-normal vision. Participants gave informed and written consent and the experiment was conducted in accordance with the protocols approved by the Human Research Ethics Advisory Panel in the School of Psychology, UNSW Sydney. All participants were naïve to the purposes of the study.

\section{Apparatus}

Participants completed the experiment in one of three similar testing cubicles. In each cubicle, visual stimuli were presented on a Display++ LCD monitor (Cambridge Research
Systems, Kent, UK) with a spatial resolution of $1920 \times 1080$ pixels, temporal resolution of $120 \mathrm{~Hz}$, mean luminance of $60 \mathrm{~cd} / \mathrm{m}^{2}$, a linear relationship between graphics card signal and luminance, and a 10-bits per pixel luminance output resolution. Participants viewed the monitor, in an otherwise darkened cubicle, from a distance of $54 \mathrm{~cm}$, for a total visual angular subtense of approximately $73.73^{\circ} \times 41.48^{\circ}$. The experiment was implemented using PsychoPy (Peirce, 2007, 2008), and the implementation and analysis made use of functionality provided by NumPy and SciPy (van der Walt, Colbert, \& Varoquaux, 2011). The code, data, and a reproducible computing environment for this study are available at https://doi.org/10.24433/Co.5eb70f0a $-1152-491 e-8 b 1 d-99477 a 23 d 562$ for Experiment 1 and at https: //doi.org/10.24433/CO.c6d54a86 $-e 380-4 d 5 c-b a 9 e-97 a 7159 f 244 f$ for Experiment 2.

\section{Stimuli}

Stimuli were constructed from renderings of a cylinder with orthographic projection, with rendering performed using Mitsuba (version 0.5.0; https://www.mitsuba -renderer.org/). Examples of single frames are shown in Figure 2, and animations are shown in Supplementary Videos 1 and 2.

The cylinder was aligned with the horizontal axis and vertically centred, and was sized to have horizontal extents of 140 pixels (Experiment 1) or 280 pixels (Experiment 2) and a vertical extent of 164 pixels. In a given rendering, the reflectance across the surface of the cylinder was determined by a texture map drawn from a pre-generated bank. Each texture map was generated from a uniform image of $50 \%$ reflectance with a central square region $(14 \times 14$ pixels $)$ containing a reflectance specific to the texture map. This central square reflectance varied across renderings between $0 \%$ to $100 \%$ reflectance in $0.5 \%$ increments. Together, these 201 texture maps formed the set of reflectances for the uniform stimulus conditions. For each experiment, we created an additional 10 sets of texture maps which included surface markings of $0 \%$ reflectance. Such surface markings were created by randomly positioning 200 ellipses, each with a random orientation, size (between 3 and 4 pixels in Experiment 1, between 1 and 9 pixels in Experiment 2), and aspect ratio (between 0.25 and 0.75 in Experiment 1, between 0.25 and 0.85 in Experiment 2). The purpose of such random variation in marking characteristics was to disrupt static textural cues to shape (Yuille \& Bülthoff, 1996). See Figure 2 for an example of the markings applied to the cylinder surfaces.

For each texture map, we rendered a sequence of $180 \mathrm{im}-$ ages in which the cylinder rotated about the horizontal axis. The extent of rotation was $\pm 5^{\circ}$ (Experiment 1 ) or $\pm 10^{\circ}$ (Experiment 2), with the degree of rotation following a sinusoidal profile over the 180 images. Such renderings were 
A

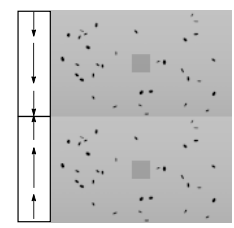

D

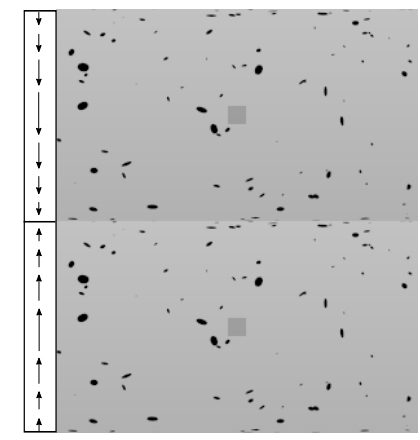

B

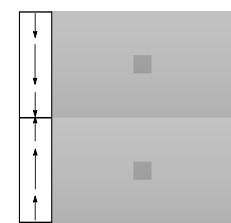

E

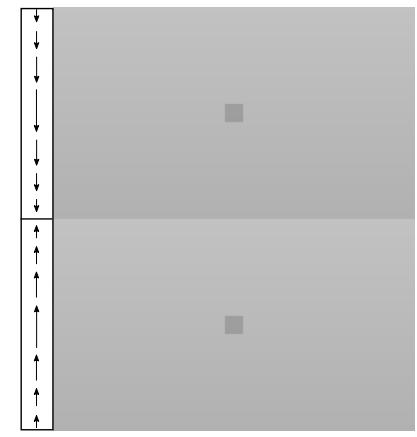

C
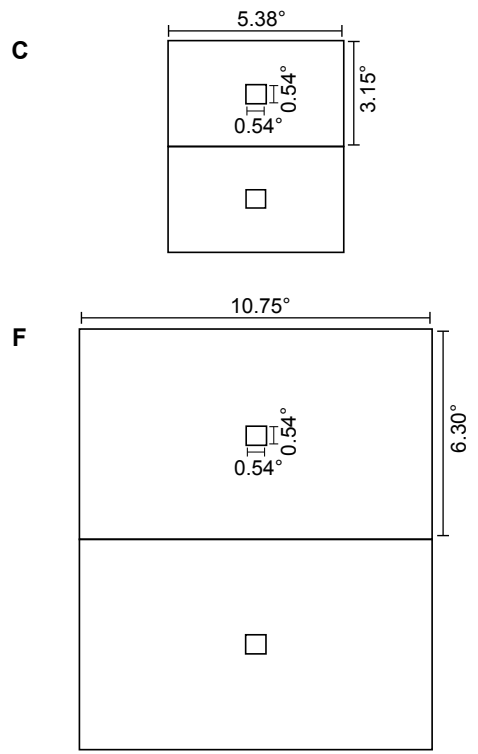

Figure 2. Stimulus examples and presentation geometry. Panels $\mathbf{A}$ and $\mathbf{B}$ show examples from the textured and uniform surface conditions, respectively, in Experiment 1 (truncated cylinders). Panels $\mathbf{D}$ and $\mathbf{E}$ show examples from the textured and uniform surface conditions, respectively, in Experiment 2 (half-cylinders). Panels $\mathbf{C}$ and $\mathbf{F}$ show the stimulus dimensions, in degrees of visual angle, from Experiments 1 and 2, respectively. The vector plots attached to the stimulus images represent approximate changes in velocity across the surfaces (not to scale).

generated with ambient illumination only, giving 11 (texture maps) $\times 201$ (central square reflectances) $\times 180$ (cylinder rotations) surface reflectance images.

For Experiment 1, we also rendered the cylinder with a uniform reflectance and non-ambient illumination to obtain a shading image. We positioned a directional light source such that the shading profile within the central 82 pixels of the cylinder had an approximately linear falloff. For Experiment 2 , we computed a synthetic shading image in which the shading profile had a linear falloff across the vertical extent of the cylinder.

The reflectance and shading images were synthesised and composited to produce the stimuli presented to participants. For Experiment 1, the desired reflectance image was first cropped to the central 82 pixels across the vertical extent of the cylinder. Ambient and scaling components were then applied to the shading image such that the multiplication with the reflectance image would produce a modulation of approximately $10 \%$ about the mean luminance of the monitor for the $50 \%$ reflectance sections. The shading image was vertically inverted to simulate illumination from below, if required. After the multiplication of the reflectance and shading images, two cylinders were composited such that they were vertically abutting in the image. The two cylinders were from the same texture set, but could have different central square reflectances and had were in temporal anti-phase (such that both rotated inward and outward in synchrony). This produced opposite directions of image motion at the surface intersection, which further promotes the impression of 3D structure (Thompson, Kersten, \& Knecht, 1992; Froyen, Feldman, \& Singh, 2013).

When presented on the monitors used in the experiments, the visible section of each cylinder was $5.38^{\circ} \times 3.15^{\circ}$ in Experiment 1 and $10.75^{\circ} \times 6.30^{\circ}$ in Experiment 2 (where degrees is in units of angular subtense). The central patches were $0.54^{\circ}$ in both experiments. Such stimulus geometries are depicted in Figure $2 \mathrm{C}$ and Figure 2F.

\section{Design and Procedure}

Both experiments used a two-way between-subjects design with factors of surface texture (uniform or textured) and surface motion (static or moving). In the uniform/static condition, the surfaces do not contain any markings and are stationary. In the uniform/moving condition, the surfaces do not contain any markings and they are in motion. In the textured/static condition, the surfaces contain markings and are stationary. In the textured/motion condition, the surfaces contain markings and are in motion. This textured/motion condition is the only in which the kinetic depth effect is expected to be strongly elicited. Participants were allocated to one of these four conditions, pseudorandomly such that each condition would have equal numbers of participants. 
There were four within-subjects manipulations that were common across each of the between-subjects conditions. The direction of the sawtooth luminance profile could be either increasing or decreasing from top to bottom (see the stimuli in Figure 2 for examples of "decreasing"), and the location of the patch of fixed reflectance (reference) could be either on the upper or the lower surface. The inclusion of such within-subjects manipulations was designed to limit the adaptation that would be present if trials were identical except for the reflectance of the comparison patch, and to balance the screen locations of the reference and comparison patches across trials.

Participants completed the experiment in one session which lasted approximately one hour. The participant's dominant eye was first determined using the 'card test' test as described by Ehrenstein, Arnold-Schulz-Gahmen, and Jaschinski (2005). Based on the outcome of this test, an occluder attached to the chinrest was adjusted such that the participant viewed the monitor with their dominant eye only. The purpose of viewing the stimulus monocularly was to eliminate the influence of conflicting stereo cues to surface shape.

Participants were then introduced to the task via a set of on-screen instructions. In Experiment 1, the instructions were passive and participants were given the opportunity to have them repeated before commencing the experiment. In Experiment 2, we incorporated a set of practice trials into the instructions in an attempt to reduce the number of participants required to be excluded due to apparent misunderstandings of task requirements.

The experiments each consisted of four runs, with a mandatory rest break between each run and halfway through each run. Each run consisted of 136 trials, where each trial contained the presentation of a single stimulus sequence (1.5 seconds) followed by the behavioural judgement. For participants in the surface motion conditions, the presentation sequence depicted one complete cycle of the cylinder's backand-forth rotation. For participants in the surface static conditions, the presentation only showed the first frame of the cylinder's rotation (in which the central patch was vertically centred). On each trial, the reflectance of one of the patches (the reference patch) was always $35 \%$.

Participants were asked to judge the relative brightness of the central squares on the two cylinders on each trial ("Was the patch on the top or bottom of the stimulus brighter? Press the up key for top or the down key for bottom"). We chose to use the term 'brightness', rather than 'lightness', to provide a stronger test of auxiliary influences (Adelson, 1993) and to simplify the task for our naïve observers. Consistent with the approach of previous studies (Adelson, 1993; Boyaci, Fang, Murray, \& Kersten, 2007), we interpret such brightness responses as implicitly relating to perceived surface reflectance (lightness).

The first four trials at the start of each run and after the halfway break of each run were practice trials, in which the reflectance of the comparison patch was randomly selected from a range approximately 5\% above and below the reference reflectance. Each run also included eight randomly-interspersed 'catch' trials, in which the comparison reflectance was $10 \%$ or $90 \%$. For the remaining 120 trials in a run, there were 30 trials for each combination of gradient direction (luminance increasing or decreasing from top to bottom) and the location of the patch of fixed reflectance (upper or lower cylinder), presented in random order. The reflectance of the comparison patch on each of these trials was determined using a Psi adaptive staircase procedure (Kontsevich \& Tyler, 1999), with separate staircases for the four combinations of gradient direction and reference patch location. As part of the staircase procedure, participants' responses were modelled using a logistic psychometric function, as given by Kingdom and Prins (2010):

$$
\psi(x ; \alpha, \beta, \gamma, \lambda)=\gamma+(1-\gamma-\lambda)\left(\frac{1}{1+e^{-\beta(x-\alpha)}}\right)
$$

This equation describes the probability of a participant selecting the comparison as the brighter patch for a given comparison reflectance $(x)$, where $\alpha$ is the point of subjective equality (PSE), $\beta$ is the slope of the psychometric function, and $\gamma$ and $\lambda$ (the guess and lapse rates) were fixed at 0.05 . The candidate comparison reflectances and PSEs were 201 values equally spaced between $0 \%$ and $100 \%$, and the candidate slopes were 50 logarithmically spaced values between 1 and 200.

\section{Analysis}

Participant summaries. One participant in each of experiment was excluded from further analysis due to an incomplete experiment session (computer malfunction in Experiment 1, time constraints in Experiment 2). Additional participants were collected as replacement.

We first inspected participant performance on the catch trials, in which they compared the relative brightness of patches with $10 \%$ or $90 \%$ reflectance against the reference reflectance of $35 \%$. Because such a difference is sufficiently large to overwhelm any perceptual change in relative appearance, we consider any 'incorrect' responses (responding that the $10 \%$ reflectance patch was brighter than the $35 \%$ patch, or that the $35 \%$ patch was brighter than the $90 \%$ patch) to indicate a lapse or task misunderstanding by the participant. We set the exclusion criterion for catch trial performance to be $80 \%$ accuracy (on both the $10 \%$ and the $90 \%$ reflectance catch trials), and $83.1 \%$ (123) and $87.7 \%$ (64) participants met this criterion in Experiments 1 and 2 respectively. Additional participants were collected as replacement for those excluded due to catch trial performance.

We next summarised participant performance on the experiment trials ( 480 per participant). The data from each trial 

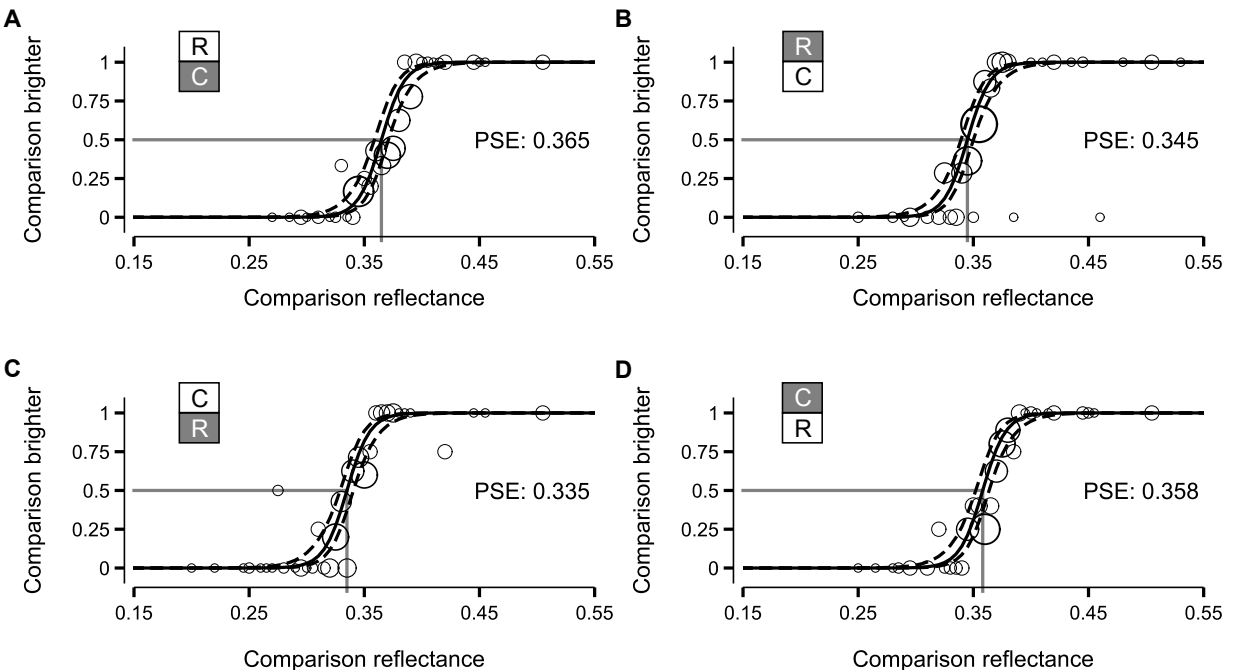

Figure 3. Example psychometric functions from a participant in the uniform/static condition in Experiment 1 (truncated cylinders). Panels show the within-subjects manipulations of luminance profile direction (increasing top to bottom in $\mathbf{A}$ and $\mathbf{C}$ and decreasing top to bottom in $\mathbf{B}$ and $\mathbf{D}$ ) and location of the reference patch (upper surface in $\mathbf{A}$ and $\mathbf{B}$, lower surface in $\mathbf{C}$ and $\mathbf{D}$ ). A key to these manipulations is provided on each panel, where the white and grey regions depict the sign of the perceptual differences under the traditional effect and " $R$ " and " $C$ " denote the locations of the reference and comparison patches, respectively. The open circles represent data points, the solid line is the fitted psychometric function, and the dashed lines are the $95 \%$ confidence interval.

indicated the reflectance value of the comparison patch and whether the participant judged the comparison as brighter than the reference patch. We used the logistic psychometric function shown in Equation 1 to capture participant performance, with separate PSE $(\alpha)$ parameters for each of the four pairwise combinations of gradient direction and reference patch location and a common slope $(\beta)$ parameter. The guess $(\gamma)$ and lapse $(\lambda)$ rates were fixed according to the participant's performance on the catch trials (Prins, 2012). Parameters of the psychometric function were obtained using a minimisation procedure which estimated the maximum likelihood of the observed data. An example of fitted psychometric functions for an example participant are shown in Figure 3 and for all participants in Supplementary Material. We found, through posthoc inspection, that we were unable to obtain reasonable parameter estimates through this method for three and four participants in Experiments 1 and 2, respectively (see Supplementary Material for a depiction). We excluded such participants from further analysis, and collected additional participants as replacement.

Finally, we condensed each participant's four PSE estimates into a single score that was indicative of their susceptibility to perceiving the two patches as differing in brightness (see Supplementary Material for tabulated mean PSEs and graphical comparisons of within-subject PSEs). We calculated the log ratio of the PSE and the reflectance of the fixed patch (Knill \& Kersten, 1991); with the PSE in the numerator when the comparison patch was located in the perceptually darker region and in the denominator when the comparison patch was located in the perceptually brighter region (where 'darker' and 'brighter' are according to the conventional sawtooth effect). The logic of this measure is that if the two regions of equal luminance in the stimulus appeared to differ in brightness, a patch located in the darker region of the stimulus would appear darker than the patch located in the brighter region of the stimulus (Arend, Buehler, \& Lockhead, 1971). When the comparison patch is located in the darker region of the stimulus, it requires a higher reflectance value to be perceived as equally bright as the reference patch. Conversely, the comparison patch requires a lower reflectance value when located in the brighter region to be perceived as equally bright as the reference patch.

We took the average of the four scores as the summary of the participant's data that was entered into group analysis. This average score indexes the extent to which the reference and comparison patches were perceived differently, in accordance with the conventional sawtooth effect. Values close to zero indicate that the reference and comparison patches were perceived as similar in brightness.

Hypothesis testing. We hypothesised that participants brightness judgements would be affected by shape information, such that the strength of the apparent brightness dif- 
ference would be reduced when the surfaces were perceived to be curved due to kinetic depth. As such, we expected participants' scores in the textured/motion condition to be lower than the scores in the other three conditions. To assess this hypothesis, we conducted planned contrasts that compared scores in the textured/motion condition to scores in the other three conditions in accordance with the method described in Furr and Rosenthal (2003) and report effect sizes as $r_{c}$ (Rosnow, Rosenthal, \& Rubin, 2000).

We used a sequential approach in the analysis of both experiments. This approach allows for the stopping of data collection at an interim analysis if there is sufficient support for the hypothesis or if such support was unlikely to be obtained with complete data collection (see Lakens (2014) for details). We were prepared to collect a maximum of 120 participants in each experiment (following exclusions), and we planned to conduct the interim analysis at the halfway point (with 60 participants). To control for the inflation of the false-positive rate with the two data inspections, we set the critical $p$ for claiming statistical significance to .025 at the interim analysis and to .034 at the final analysis (Lakens, 2014). The reported $p$ values are uncorrected for the sequential approach.

\section{Results and Discussion}

We hypothesised that the typical perceptual effect of two identical and abutting luminance ramps, in which the ramps appear to be of unequal brightness, would be diminished if the kinetic depth effect indicated that the ramps were reflected from two curved surfaces. Consistent with this hypothesis, a planned contrast indicated that the strength of the apparent brightness difference was significantly lower in the textured-motion condition in comparison with the aggregate of the textured-static, uniform-motion, and uniformstatic conditions $\left(t(116)=3.96, p<.001, r_{c}=0.35\right)$. Exploration of the strength of the apparent brightness difference across conditions, shown in Figure 4, shows that the effect was close to zero $(M=0.015,95 \%$ CI $[0.008,0.021])$ when surface texture markings facilitated the kinetic depth effect during cylinder rotation. This was much lower than the apparent brightness difference produced in the traditional stimulus without surface markings and with static presentation $(M=0.040,95 \%$ CI $[0.028,0.053])$. The reduction in the apparent brightness difference does not seem to be due to the presence of the surface texture markings, as a strong apparent brightness difference was evident with textured static presentation $(M=0.032,95 \%$ CI $[0.024,0.041])$. It does also not seem to be due to the motion of the central square patches, which produced a strong apparent brightness difference in the absence of surface markings ( $M=0.041,95 \% \mathrm{CI}$ $[0.032,0.052])$.

Hence, shape information derived from the kinetic depth effect appears capable of influencing judgments of lightness. This provides evidence in support of the cue invariance of

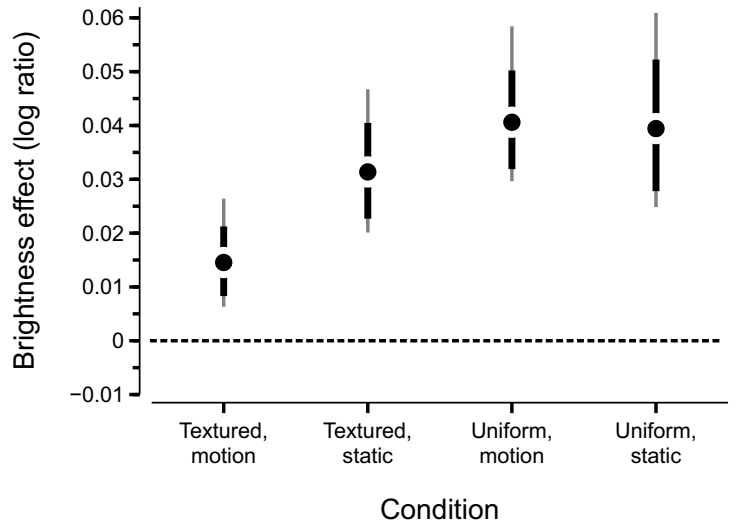

Figure 4. Strength of the apparent brightness difference for each of the four presentation conditions in Experiment 1 (trucated cylinders). Points show the means across participants and the black and grey lines show the $95 \%$ and $99 \%$ confidence intervals, respectively.

the relationship between shape and lightness (Buckley et al., 1994), suggesting that such coupling is robust to the specific cue that provides shape information. The influence of kinetic depth obtained here also reinforces the general importance of shape to the perception of surface material (Marlow, Todorović, \& Anderson, 2015; Marlow \& Anderson, 2015, 2016).

The results presented here suggest that the neural representations of surface reflectance are capable of interacting with those pertaining to surface shape from kinetic depth. The neural mechanisms for the kinetic depth effect are typically associated with the dorsal pathway (Andersen \& Bradley, 1998; Bradley, Chang, \& Andersen, 1998; Farivar, 2009; Grunewald, Bradley, \& Andersen, 2002), although there is also reported activation of areas within the ventral pathway (Kriegeskorte et al., 2003). The neural mechanisms of lightness are unclear (Kingdom, 2011; Roe et al., 2012), with reported correlates within each of the ventral pathway (Kingdom, 2011; Roe et al., 2012), the dorsal pathway (Perna, Tosetti, Montanaro, \& Morrone, 2005), and lower-level visual areas (Anderson, Dakin, \& Rees, 2009; Boyaci et al., 2007; Boyaci, Fang, Murray, \& Kersten, 2010; Roe, Lu, \& Hung, 2005). An intriguing possibility is that representations in low-level visual areas may tag surface properties such as reflectance and shape, in a manner reminiscent of the "intrinsic image" approach in computer vision (Barrow \& Tenenbaum, 1978). Interestingly, V1 appears to receive feedback signals from MT relating to shape from kinetic depth (Grunewald et al., 2002), which provides a potential mechanism by which shape from kinetic depth could interact with calculations concerning surface reflectance under this scenario.

Although the results of this experiment demonstrate that 
brightness can be affected by shape from kinetic depth, our findings differ from the reported abolishment of lightness differences with contour cues to shape (Knill \& Kersten, 1991) in that the differences were reduced, rather than eliminated, in our study. However, such an incomplete reduction has also been reported with contours and with shape from stereo cues (Buckley et al., 1994). A distinction of potential importance between Buckley et al. (1994), and the current study, and Knill and Kersten (1991) relates to the nature of the task. While participants were instructed to make their judgements based on lightness ('surface colour') in Knill and Kersten (1991), we and Buckley et al. (1994) instructed participants to make their judgements based on brightness (see the Design and Procedure for the rationale of using a brightness task in the current study). Although "border effects", such as the sawtooth profile used here, tend to produce experiences of lightness and brightness that are highly correlated, it is possible that a brightness difference may remain in scenarios in which there is no lightness difference.

Buckley et al. (1994) also speculated that a mismatch between the surface curvature and the sawtooth luminance profile may have been responsible for the residual brightness difference that they reported. Similarly, we wondered whether our use of truncated cylinders may have weakened the kinetic depth effect such that the surface curvature was not reliably perceived by all participants. Our rationale for using truncated cylinders was that their geometry permits an approximately linear luminance ramp when illuminated by a single directional light source. However, a limitation of the geometry is that it reduces the extent of curvature and hence the magnitude of velocity differences that underlie the kinetic depth effect. Inspection of the distribution of apparent brightness differences across participants (shown in Supplementary Figure 1) suggested that a small subset of participants experienced strong effects, leading to the relatively small elevation of the mean above zero.

With the aim of replicating the effect of shape kinetic depth on lightness while attempting to increase the strength of the kinetic depth cue, we conducted a second experiment using the half-cylinders shown in Figure 2. Because the halfcylinder geometry does not allow a linear luminance ramp to be produced from a simple lighting arrangement, we applied a synthetic linear illumination ramp rather than rendering a complex but physically realistic scene. Apart from the changes to the stimuli, all other aspects of the experimental design and procedure remained the same as the first experiment.

Consistent with the results from the first experiment, a planned contrast indicated that the strength of the apparent brightness difference was significantly lower in the kinetic depth condition in comparison with these other three conditions $\left(t(56)=3.18, p=.001, r_{c}=0.39\right)$. As shown in Figure 5, the apparent brightness difference was again not

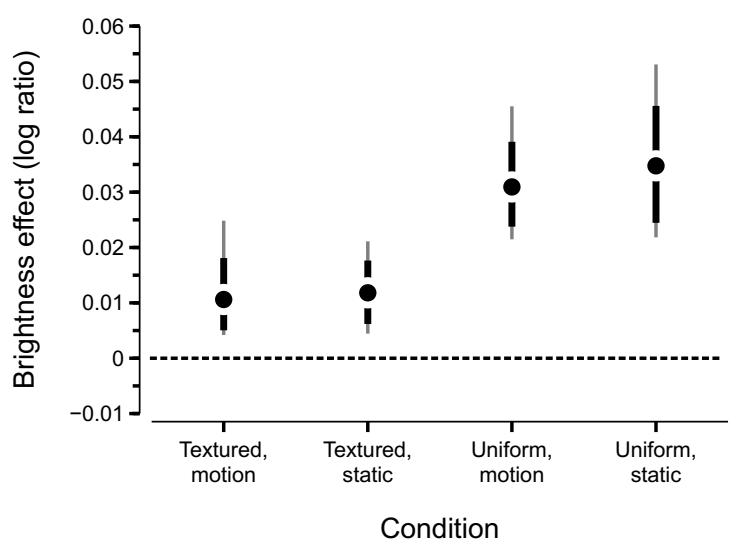

Figure 5. Strength of the apparent brightness difference for each of the four presentation conditions in Experiment 2 (half-cylinders). Points show the means across participants and the black and grey lines show the $95 \%$ and $99 \%$ confidence intervals, respectively.

abolished in the kinetic depth condition $(M=0.011,95 \% \mathrm{CI}$ $[0.005,0.018])$. Thus, it seems that this residual brightness difference is not accounted for by the relative strength of the impression of shape from kinetic depth. The impression of surface curvature from binocular disparity (Buckley et al., 1994) and kinetic depth may be less capable than border contours (Knill \& Kersten, 1991) of affecting lightness estimates, potentially both within and across individuals. However, given that an incomplete reduction in brightness difference has also been reported with contours (Buckley et al., 1994), it remains unclear to what extent shape cues are capable of abolishing the brightness differences associated with the sawtooth luminance profile.

An additional change in the results of the second experiment, in comparison with the results of the first experiment, is that the apparent brightness difference was also reduced in the condition with surface markings but static presentation $(M=0.012,95 \% \mathrm{CI}[0.006,0.018])$. We consider this difference is likely to be attributable to the increased availability of static textural cues to curvature with the half-cylinders of the second experiment (compare Figure 2A and Figure 2D). These additional static shape cues may have caused participants in the static texture condition to perceive the two surfaces as curved, leading to a reduction in apparent brightness differences via a similar mechanism as shape from contour (Knill \& Kersten, 1991), binocular disparity (Buckley et al., 1994), and kinetic depth (the previous experiment). Although we attempted to disrupt the influence of texture cues to surface shape by creating variation in the texture markings applied to the surfaces in the stimulus, we may not have been able to completely remove the effects of static cues in the second experiment. Hence, the results of the 
second experiment do not, in isolation, provide support for an influence of kinetic depth on lightness. However, given the results of the first experiment, we consider it likely that kinetic depth can influence lightness and that the results of the second experiment demonstrate an additional role for static textural cues to shape in lightness-which is consistent with the findings of Marlow and Anderson (2016).

The results of this study are consistent with the visual system using a representation of curvature, identified from motion, in determining surface lightness. However, it is possible that an explicit representation of curvature may not be necessary for lightness to be affected by surface motionthere may instead be a heuristic (Kingdom, 2008; Ramachandran, 1985) that relates particular moving and static factors to lightness or illumination without direct reference to shape. For example, Kingdom (2008) proposed a heuristic in which luminance gradients that covary with changes in apparent shape (surface orientation) are attributed to shading (illumination); perhaps an alternate form of this heuristic could be applied in which luminance gradients that covary with changes in texture velocity are attributed to shading. However, the robustness and general applicability of such a heuristic is unclear. Furthermore, a shape-unaware heuristic that attributes luminance gradients to shading would be limited in its ability to contribute contextual information regarding scene illumination (such as the light source position).

Overall, the aim of this study was to determine whether shape information provided by motion can be used to inform judgements of surface lightness. We found that the shape information provided by the kinetic depth effect was capable of affecting apparent surface lightness, with a sawtooth luminance profile perceived as being composed of surfaces that were more similar in reflectance when motion cues were indicative of surface curvature. This is consistent with the cue-invariance of the interaction between shape and lightness, and indicates that the human visual system can use evidence from motion to evaluate competing hypotheses regarding surface material.

\section{References}

Adelson, E. H. (1993). Perceptual organization and the judgment of brightness. Science, 262(5142), 2042-2044.

Andersen, R. A., \& Bradley, D. C. (1998). Perception of three-dimensional structure from motion. Trends in Cognitive Sciences, 2(6), 222-228.

Anderson, E. J., Dakin, S. C., \& Rees, G. (2009). Monocular signals in human lateral geniculate nucleus reflect the Craik-Cornsweet-O'Brien effect. Journal of Vision, 9(12), 14.1-1418.

Arend, L. E., Buehler, J. N., \& Lockhead, G. R. (1971). Difference information in brightness perception. Perception and Psychophysics, 9(3), 367.
Barrow, H. G., \& Tenenbaum, J. M. (1978). Recovering intrinsic scene characteristics from images. In A. Hanson \& E. Riseman (Eds.), Computer vision systems. Artificial Intelligence Center, SRI International Menlo Park, CA.

Bergström, S. S. (1977). Common and relative components of reflected light as information about the illumination, colour, and three-dimensional form of objects. Scandinavian Journal of Psychology, 18, 180-186.

Boyaci, H., Fang, F., Murray, S. O., \& Kersten, D. (2007). Responses to lightness variations in early human visual cortex. Current Biology, 17(11), 989-993.

Boyaci, H., Fang, F., Murray, S. O., \& Kersten, D. (2010). Perceptual grouping-dependent lightness processing in human early visual cortex. Journal of Vision, 10(9), 112.

Bradley, D. C., Chang, G. C., \& Andersen, R. A. (1998). Encoding of three-dimensional structure-from-motion by primate area MT neurons. Nature, 392(6677), 714717.

Buckley, D., Frisby, J. P., \& Freeman, J. (1994). Lightness perception can be affected by surface curvature from stereopsis. Perception, 23(8), 869-881.

Cornsweet, T. (1970). Vision perception. New York: Academic Press.

Craik, K. J. (1966). The nature of psychology. New York: Cambridge University Press.

Ehrenstein, W. H., Arnold-Schulz-Gahmen, B. E., \& Jaschinski, W. (2005). Eye preference within the context of binocular functions. Graefe's Archive for Clinical and Experimental Ophthalmology, 243, 926-932.

Fang, F., \& He, S. (2004). Stabilized structure from motion without disparity induces disparity adaptation. Current Biology, 14, 247-251.

Farivar, R. (2009). Dorsal-ventral integration in object recognition. Brain Research Reviews, 61(2), 144-153.

Freeman, R. D. (1998). Binocular vision: the neural integration of depth and motion. Current Biology, 8, R761R764.

Froyen, V., Feldman, J., \& Singh, M. (2013). Rotating columns: relating structure-from-motion, accretion/deletion, and figure/ground. Journal of Vision, 13.

Furr, R. M., \& Rosenthal, R. (2003). Repeated-measures contrasts for "multiple-pattern" hypotheses. Psychological Methods, 8, 275-293.

Gilchrist, A. (1977). Perceived lightness depends on perceived spatial arrangement. Science, 195(4274), 185187.

Gilchrist, A. (1980). When does perceived lightness depend on perceived spatial arrangement? Perception \& Psychophysics, 28(6), 527-538.

Grunewald, A., Bradley, D. C., \& Andersen, R. A. (2002). 
Neural correlates of structure-from-motion perception in macaque V1 and MT. Journal of Neuroscience, 22(14), 6195-6207.

Kersten, D., Bülthoff, H. H., Schwartz, B. L., \& Kurtz, K. J. (1992). Interaction between transparency and structure from motion. Neural Computation, 4(4), 573-589.

Kersten, D., Mamassian, P., \& Yuille, A. (2004). Object perception as Bayesian inference. Annual Review of Psychology, 55, 271-304.

Kersten, D., \& Yuille, A. (2003). Bayesian models of object perception. Current Opinion in Neurobiology, 13(2), $150-158$.

Kham, K., \& Blake, R. (2000). Depth capture by kinetic depth and by stereopsis. Perception, 29, 211-220.

Kingdom, F. A. A. (2008). Perceiving light versus material. Vision Research, 48, 2090-2105.

Kingdom, F. A. A. (2011). Lightness, brightness and transparency: a quarter century of new ideas, captivating demonstrations and unrelenting controversy. Vision Research, 51(7), 652-673.

Kingdom, F. A. A., \& Moulden, B. (1988). Border effects on brightness: a review of findings, models and issues. Spatial Vision, 3(4), 225-262.

Kingdom, F. A. A., \& Prins, N. (2010). Psychophysics: A practical introduction. London: Academic Press.

Knill, D. C., \& Kersten, D. (1991). Apparent surface curvature affects lightness perception. Nature, 351(6323), 228-230.

Kontsevich, L. L., \& Tyler, C. W. (1999). Bayesian adaptive estimation of psychometric slope and threshold. Vision Research, 39, 2729-2737.

Kriegeskorte, N., Sorger, B., Naumer, M., Schwarzbach, J., van den Boogert, E., Hussy, W., \& Goebel, R. (2003). Human cortical object recognition from a visual motion flowfield. Journal of Neuroscience, 23(4), 14511463.

Lakens, D. (2014). Performing high-powered studies efficiently with sequential analyses. European Journal of Social Psychology, 44(7), 701-710.

Marlow, P. J., \& Anderson, B. L. (2015). Material properties derived from three-dimensional shape representations. Vision Research, 115, 199-208.

Marlow, P. J., \& Anderson, B. L. (2016). Motion and texture shape cues modulate perceived material properties. Journal of Vision, 16, 5.

Marlow, P. J., Todorović, D., \& Anderson, B. L. (2015). Coupled computations of three-dimensional shape and material. Current Biology, 25(6), R221-R222.

Nayar, S. K., \& Bolle, R. M. (1996). Reflectance based object recognition. International Journal of Computer Vision, 17(3), 219-240.

Nieder, A. (2003). Interrelation of kinetic and stereoscopic depth: behavior and physiology in vertebrates. Be- havioural Processes, 64, 13-16.

O'Brien, V. (1958). Contour perception, illusion and reality. Journal of the Optical Society of America, 48(2), 112119.

O’Toole, A. J., Vetter, T., \& Blanz, V. (1999). Threedimensional shape and two-dimensional surface reflectance contributions to face recognition: an application of three-dimensional morphing. Vision Research, 39, 3145-3155.

Peirce, J. W. (2007). PsychoPy-psychophysics software in Python. Journal of Neuroscience Methods, 162, 8-13.

Peirce, J. W. (2008). Generating stimuli for neuroscience using PsychoPy. Frontiers in Neuroinformatics, 2, 10.

Perna, A., Tosetti, M., Montanaro, D., \& Morrone, M. C. (2005). Neuronal mechanisms for illusory brightness perception in humans. Neuron, 47(5), 645-651.

Prins, N. (2012). The psychometric function: the lapse rate revisited. Journal of Vision, 12(6).

Purves, D., Shimpi, A., \& Lotto, R. B. (1999). An empirical explanation of the Cornsweet effect. Journal of Neuroscience, 19(19), 8542-8551.

Ramachandran, V. S. (1985). The neurobiology of perception. Perception, 14, 97-103.

Roe, A. W., Chelazzi, L., Connor, C. E., Conway, B. R., Fujita, I., Gallant, J. L., . . V Vanduffel, W. (2012). Toward a unified theory of visual area V4. Neuron, 74(1), 1229.

Roe, A. W., Lu, H. D., \& Hung, C. P. (2005). Cortical processing of a brightness illusion. Proceedings of the National academy of Sciences of the United States of America, 102(10), 3869-3874.

Rosnow, R. L., Rosenthal, R., \& Rubin, D. B. (2000). Contrasts and correlations in effect-size estimation. Psychological Science, 11(6), 446-453.

Russell, R., Biederman, I., Nederhouser, M., \& Sinha, P. (2007). The utility of surface reflectance for the recognition of upright and inverted faces. Vision Research, 47, 157-165.

Thompson, W. B., Kersten, D., \& Knecht, W. R. (1992). Structure-from-motion based on information at surface boundaries. Biological Cybernetics, 66, 327-333.

van den Brink, G., \& Keemink, C. (1976). Luminance gradients and edge effects. Vision Research, 16(2), 155159.

van der Walt, S., Colbert, S., \& Varoquaux, G. (2011). The NumPy array: A structure for efficient numerical computation. Computing in Science \& Engineering, 13(2), 22-30.

Wallach, H., \& O'Connell, D. N. (1953). The kinetic depth effect. Journal of Experimental Psychology, 45, 205217.

Yuille, A., \& Bülthoff, H. (1996). Bayesian decision theory and psychophysics. In D. C. Knill \& W. Richards 
(Eds.), Perception as Bayesian inference (pp. 123161). Cambridge University Press. 